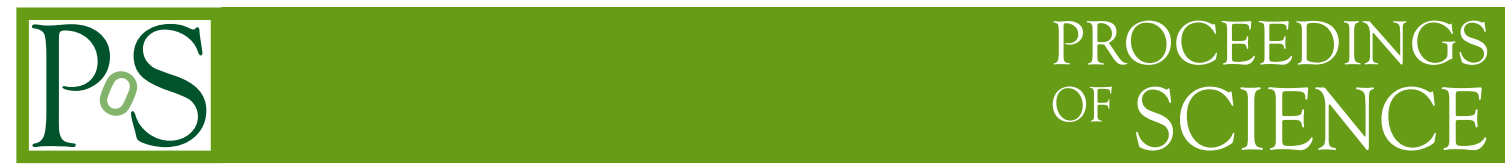

\title{
Sterile neutrino mixing now and in the next $10+$ years
}

\section{Carlo Giunti*}

INFN, Sezione di Torino, Via P. Giuria 1, I-10125 Torino, Italy

E-mail: giunti@to.infn.it

Short-baseline neutrino oscillation anomalies indicate that there may be a sterile neutrino at the $\mathrm{eV}$ scale. We briefly review the theory of neutrino oscillations in the framework of $3+1$ mixing with one sterile neutrino and the status of the global fit of short-baseline neutrino oscillation data. We also briefly discuss the sensitivities of future experiments.

Neutrino Oscillation Workshop

4 - 11 September, 2016

Otranto (Lecce, Italy)

${ }^{*}$ Speaker. 
In this review we consider the LSND [1,2], Gallium [3-7] and reactor [8-10] short-baseline neutrino oscillation anomalies and we discuss their explanation in the framework of an effective $3+1$ mixing scheme with one sterile neutrino at the eV scale (see Ref. [11]). In this framework, the interacting left-handed flavor neutrino fields $v_{e L}, v_{\mu L}, v_{\tau L}$, are unitary linear combinations of four left-handed massive neutrino fields $v_{1 L}, v_{2 L}, v_{3 L}, v_{4 L}$ with respective masses $m_{1}, m_{2}, m_{3}, m_{4}$ :

$$
v_{\alpha L}=\sum_{k=1}^{4} U_{\alpha k} v_{k L} \quad(\alpha=e, \mu, \tau)
$$

where $U$ is the $4 \times 4$ unitary mixing matrix. The oscillation probabilities of the flavor neutrinos in short-baseline experiments are given by [12]

$$
\underset{\substack{(-) \\ v_{\alpha} \rightarrow v_{\beta}}}{(\mathrm{SBL})} \simeq\left|\delta_{\alpha \beta}-\sin ^{2} 2 \vartheta_{\alpha \beta} \sin ^{2}\left(\frac{\Delta m_{41}^{2} L}{4 E}\right)\right|, \quad \text { with } \quad \sin ^{2} 2 \vartheta_{\alpha \beta}=\left.4\left|U_{\alpha 4}\right|^{2}\left|\delta_{\alpha \beta}-\right| U_{\beta 4}\right|^{2} \mid \text {, }
$$

and $\Delta m_{41}^{2}=m_{4}^{2}-m_{1}^{2}$.

Figures 1(a), 2(a), and 3(a) show the allowed regions in the $\sin ^{2} 2 \vartheta_{e \mu}-\Delta m_{41}^{2}, \sin ^{2} 2 \vartheta_{e e}-\Delta m_{41}^{2}$ and $\sin ^{2} 2 \vartheta_{\mu \mu}-\Delta m_{41}^{2}$ planes obtained in the pragmatic $3+1$ global (PrGLO) fit of short-baseline neutrino oscillation data $[11,13,14]$. These regions are relevant, respectively, for $\stackrel{(-)}{v_{\mu}} \rightarrow \stackrel{(-)}{v_{e}}$ appearance, $\stackrel{(-)}{v_{e}}$ disappearance and $\stackrel{(-)}{v_{\mu}}$ disappearance searches. Figure 1 (a) shows also the region allowed by $v_{\mu} \rightarrow \stackrel{(-)}{v_{e}}$ appearance data and the constraints from $\stackrel{(-)}{v}_{e}$ disappearance and $\stackrel{(-)}{v_{\mu}}$ disappearance data. The best-fit values of the oscillation parameters are $\left(\Delta m_{41}^{2}\right)_{\mathrm{bf}}=1.6 \mathrm{eV}^{2},\left(\left|U_{e 4}\right|^{2}\right)_{\mathrm{bf}}=0.027$, $\left(\left|U_{\mu 4}\right|^{2}\right)_{\mathrm{bf}}=0.012$, which imply $\left(\sin ^{2} 2 \vartheta_{e \mu}\right)_{\mathrm{bf}}=0.0013,\left(\sin ^{2} 2 \vartheta_{e e}\right)_{\mathrm{bf}}=0.10$ and $\left(\sin ^{2} 2 \vartheta_{\mu \mu}\right)_{\mathrm{bf}}=$ 0.050 .

Figure 3(a) shows a comparison of the allowed regions in the $\sin ^{2} 2 \vartheta_{\mu \mu}-\Delta m_{41}^{2}$ plane with the exclusion curves obtained recently by the IceCube [15] and MINOS [16] experiments. One can see that they disfavor the low- $\Delta m_{41}^{2}$ and high- $\sin ^{2} 2 \vartheta_{\mu \mu}$ part of the allowed region. This is confirmed by the results presented in Ref. [17], where the $3+1$ global fit of Ref. [18] was updated with the addition of the IceCube data.

Because of the scarcity of sensitive data, of the possible existence of unknown systematic errors, and of the appearance-disappearance tension (see Ref. [19]), the possible existence of light sterile neutrinos at the $\mathrm{eV}$ scale is controversial and needs new reliable experimental checks. Fortunately, there is an impressive program of new experiments which are planned to check the existence of eV sterile neutrinos (see the reviews in Refs. [11, 20-22]). Figures 1(b), 2(b), and 3(b) show a comparison of the sensitivities of future experiments with the corresponding allowed regions for, respectively, $\stackrel{(-)}{v}_{\mu} \rightarrow \stackrel{(-)}{v_{e}}$ transitions (SBN [23], nuPRISM [24], JSNS ${ }^{2}$ [25]), $\stackrel{(-)}{v_{e}}$ disappearance (CeSOX [26], BEST [27], IsoDAR@KamLAND [28], IsoDAR@C-ADS [29], DANSS [30], NEOS [31], Neutrino-4 [32], PROSPECT [33], SoLid [34], STEREO [35], KATRIN [36]), and $v_{\mu}^{(-)}$ disappearance (SBN [23], KPipe [37]).

Let us finally emphasize that the discovery of the existence of sterile neutrinos would be a major discovery which would have a profound impact not only on neutrino physics, but on our whole view of fundamental physics, because sterile neutrinos are elementary particles beyond the Standard Model. The existence of light sterile neutrinos would prove that there is new physics 


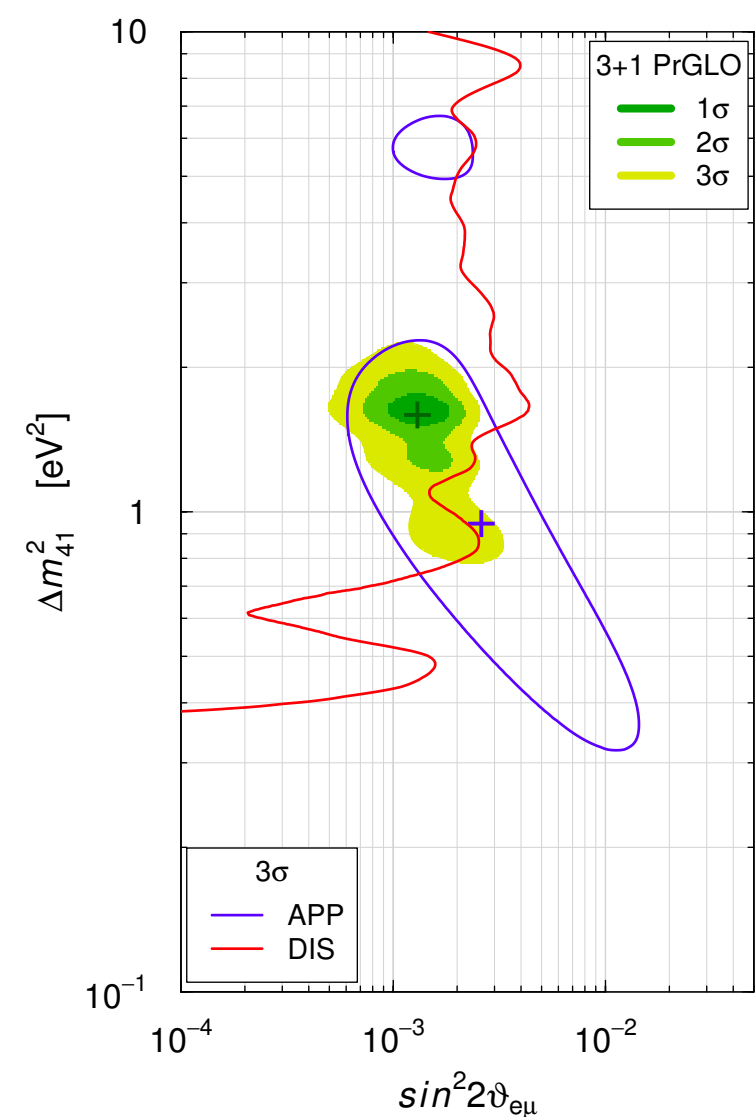

(a)

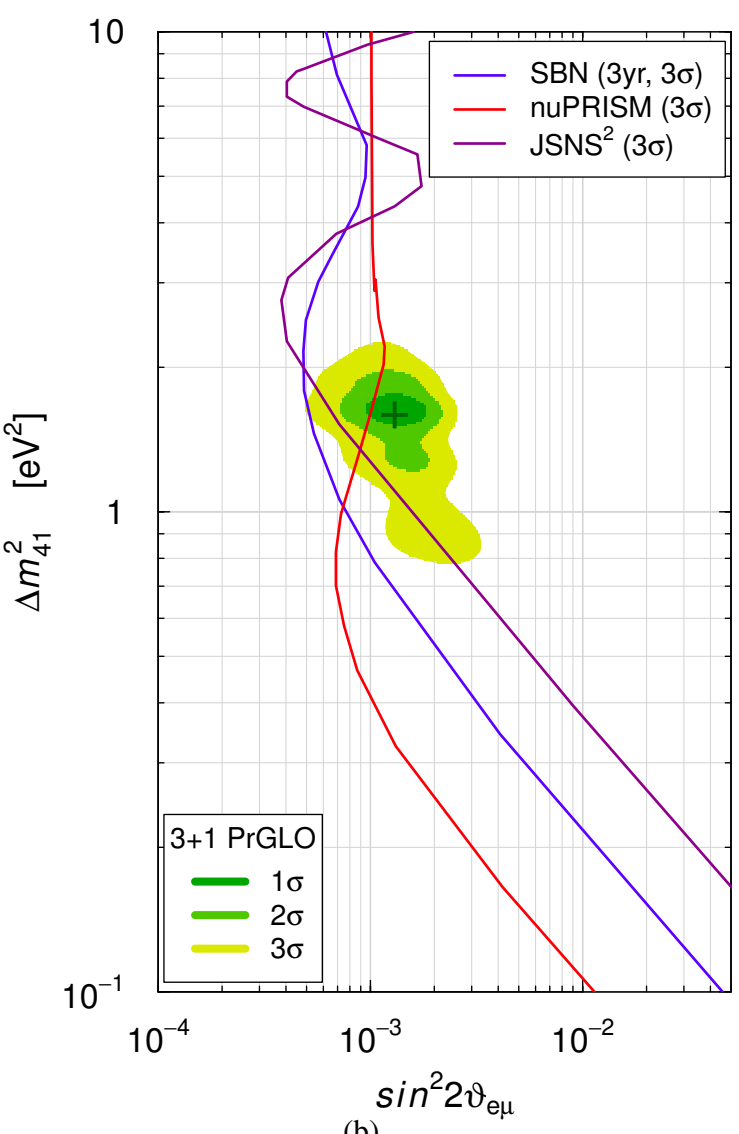

(b)

Figure 1: Allowed regions in the $\sin ^{2} 2 \vartheta_{e \mu}-\Delta m_{41}^{2}$ plane obtained in the pragmatic $3+1$ global (PrGLO) fit of short-baseline neutrino oscillation data $[11,13,14]$. (a): comparison with the $3 \sigma$ allowed regions obtained from $v_{\mu} \rightarrow \stackrel{(-)}{v_{e}}$ short-baseline appearance data (APP) and the $3 \sigma$ exclusion curve obtained from short-baseline disappearance data (DIS). (b): comparison with the sensitivities of future experiments. The best-fit points of the global (PrGLO) and APP fits are indicated by crosses.

beyond the Standard Model at low energies and their properties can give important information on this new physics.

\section{References}

[1] LSND collaboration, C. Athanassopoulos et al., Candidate events in a search for $\bar{v}_{\mu} \rightarrow \bar{v}_{e}$ oscillations, Phys. Rev. Lett. 75 (1995) 2650-2653, [nucl-ex/9504002].

[2] LSND collaboration, A. Aguilar et al., Evidence for neutrino oscillations from the observation of $\bar{v}_{e}$ appearance in a $\bar{v}_{\mu}$ beam, Phys. Rev. D64 (2001) 112007, [hep-ex/ 0104049 ].

[3] SAGE collaboration, J. N. Abdurashitov et al., Measurement of the response of a Ga solar neutrino experiment to neutrinos from an Ar-37 source, Phys. Rev. C73 (2006) 045805, [nucl-ex/0512041].

[4] M. Laveder, Unbound neutrino roadmaps, Nucl. Phys. Proc. Suppl. 168 (2007) 344-346. 


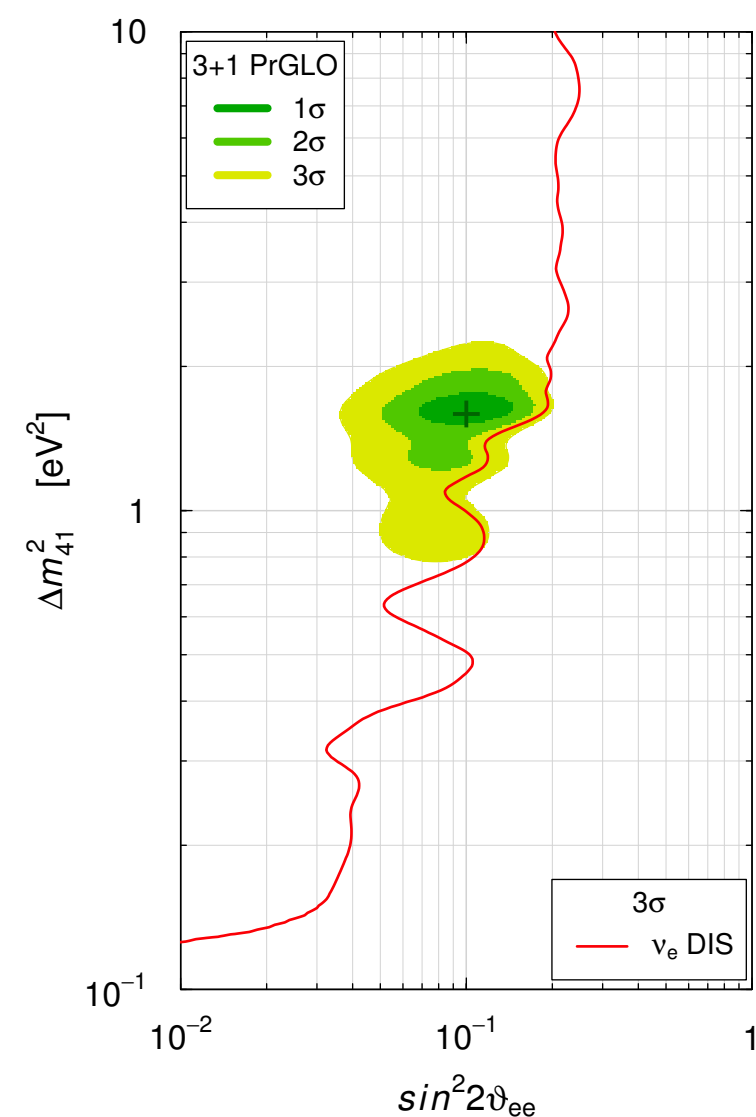

(a)

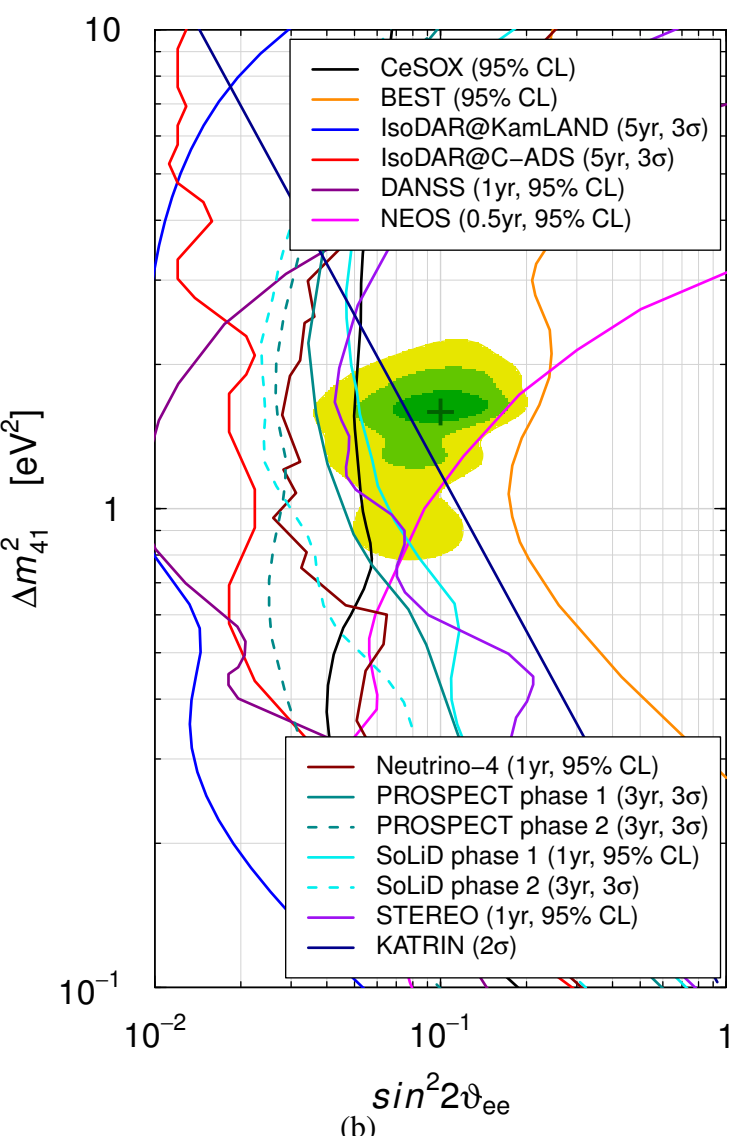

(b)

Figure 2: Allowed regions in the $\sin ^{2} 2 \vartheta_{e e}-\Delta m_{41}^{2}$ plane obtained in the pragmatic $3+1$ global (PrGLO) fit of short-baseline neutrino oscillation data $[11,13,14]$. (a): comparison with the $3 \sigma$ exclusion curve obtained from $\stackrel{(-)}{v}$ short-baseline disappearance data $\left(v_{e}\right.$ DIS), (b): comparison with the sensitivities of future experiments. The best-fit point of the global (PrGLO) fit is indicated by a cross.

[5] C. Giunti and M. Laveder, Short-Baseline Active-Sterile Neutrino Oscillations?, Mod. Phys. Lett. A22 (2007) 2499-2509, [hep-ph/0610352].

[6] C. Giunti and M. Laveder, Statistical Significance of the Gallium Anomaly, Phys. Rev. C83 (2011) 065504, [arXiv:1006.3244].

[7] C. Giunti, M. Laveder, Y. Li, Q. Liu and H. Long, Update of Short-Baseline Electron Neutrino and Antineutrino Disappearance, Phys. Rev. D86 (2012) 113014, [arXiv: 1210 . 5715].

[8] G. Mention et al., The Reactor Antineutrino Anomaly, Phys. Rev. D83 (2011) 073006, [arXiv:1101.2755].

[9] T. A. Mueller et al., Improved Predictions of Reactor Antineutrino Spectra, Phys. Rev. C83 (2011) 054615, [arXiv:1101.2663].

[10] P. Huber, On the determination of anti-neutrino spectra from nuclear reactors, Phys. Rev. C84 (2011) 024617, [arXiv:1106.0687].

[11] S. Gariazzo, C. Giunti, M. Laveder, Y. Li and E. Zavanin, Light sterile neutrinos, J. Phys. G43 (2016) 033001, [arXiv: 1507.08204$]$. 


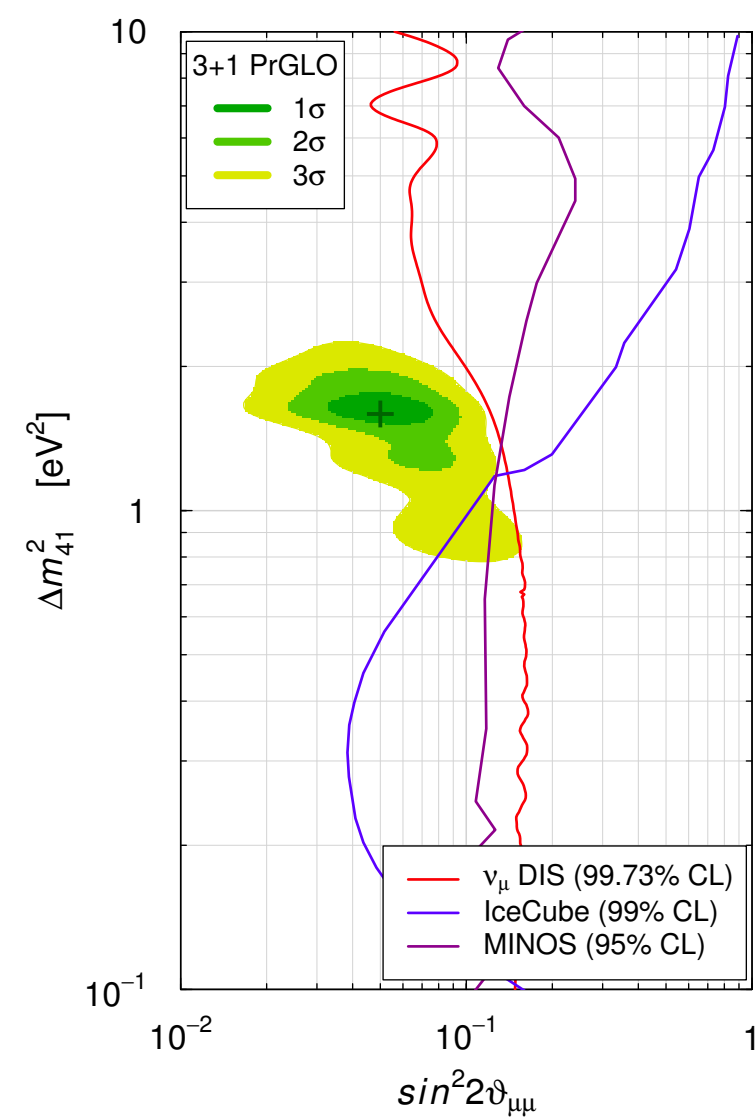

(a)

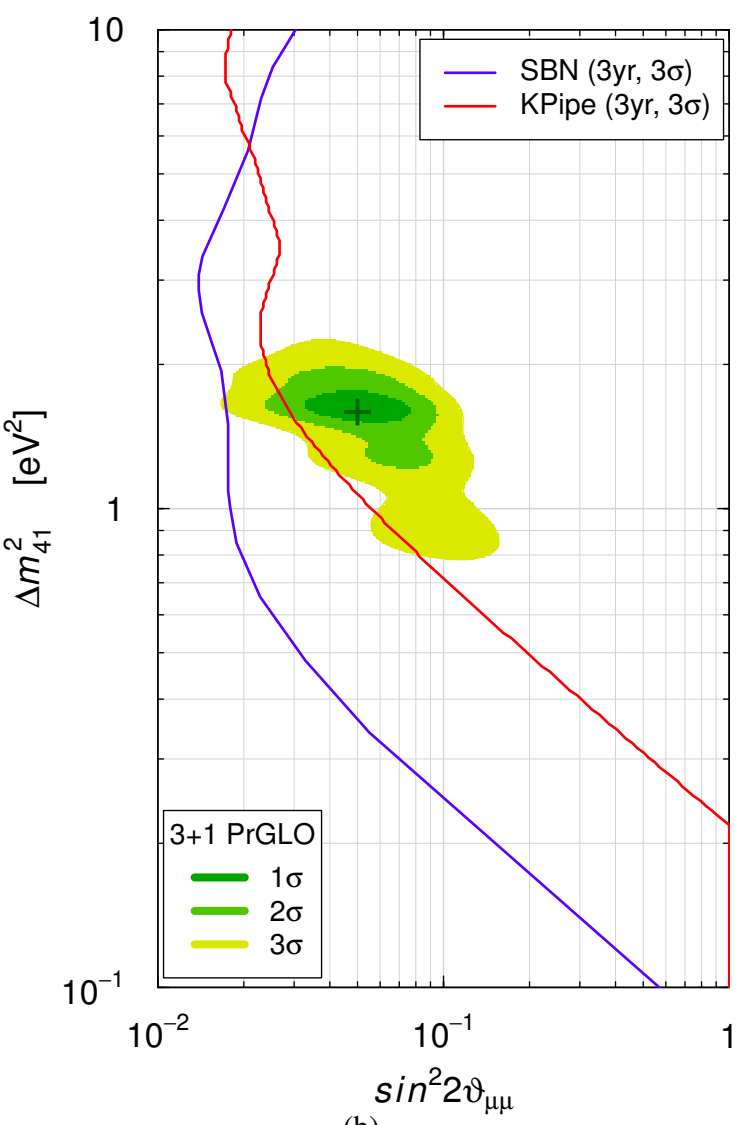

(b)

Figure 3: Allowed regions in the $\sin ^{2} 2 \vartheta_{\mu \mu}-\Delta m_{41}^{2}$ plane obtained in the pragmatic $3+1$ global (PrGLO) fit of short-baseline neutrino oscillation data $[11,13,14]$. (a): comparison with the $3 \sigma$ exclusion curve obtained from $\stackrel{(-)}{v_{\mu}}$ short-baseline disappearance data ( $v_{\mu}$ DIS), (b): comparison with the sensitivities of future experiments. The best-fit point of the global (PrGLO) fit is indicated by a cross.

[12] S. M. Bilenky, C. Giunti and W. Grimus, Neutrino mass spectrum from the results of neutrino oscillation experiments, Eur. Phys. J. C1 (1998) 247-253, [hep-ph/9607372].

[13] C. Giunti, M. Laveder, Y. Li and H. Long, A Pragmatic View of Short-Baseline Neutrino Oscillations, Phys. Rev. D88 (2013) 073008, [arXiv:1308.5288].

[14] C. Giunti, Oscillations Beyond Three-Neutrino Mixing, arXiv:1609.04688.

[15] ICECUBE collaboration, M. G. Aartsen et al., Searches for Sterile Neutrinos with the IceCube Detector, Phys. Rev. Lett. 117 (2016) 071801, [arXiv: 1605.01990$].$

[16] MINOS collaboration, P. Adamson et al., A search for sterile neutrinos mixing with muon neutrinos in MINOS, Phys. Rev. Lett. 117 (2016) 151803, [arXiv:1607.01176].

[17] G. Collin, C. Arguelles, J. Conrad and M. Shaevitz, First Constraints on the Complete Neutrino Mixing Matrix with a Sterile Neutrino, Phys. Rev. Lett. 117 (2016) 221801, [arXiv:1607. 00011$].$

[18] G. H. Collin, C. A. Arguelles, J. M. Conrad and M. H. Shaevitz, Sterile Neutrino Fits to Short Baseline Data, Nucl. Phys. B908 (2016) 354-365, [arXiv: 1602.00671$].$ 
[19] C. Giunti and E. M. Zavanin, Appearance-Disappearance Relation in $3+N_{s}$ Short-Baseline Neutrino Oscillations, Mod. Phys. Lett. A31 (2016) 1650003, [arXiv: 1508.03172$].$

[20] C. Giunti, Light Sterile Neutrinos: Status and Perspectives, Nucl. Phys. B908 (2016) 336-353, [arXiv:1512.04758].

[21] L. Stanco, Search for Sterile Neutrinos at Long and Short Baselines, arXiv: 1604.06769.

[22] A. Fava, Experimental investigation of the thriving mystery of sterile neutrinos, Rev. Phys. 1 (2016) 52-59.

[23] MicroBooNE, LAR1-ND, ICARUS-WA104 collaboration, R. Acciarri et al., A Proposal for a Three Detector Short-Baseline Neutrino Oscillation Program in the Fermilab Booster Neutrino Beam, arXiv:1503.01520.

[24] NUPRISM collaboration, S. Bhadra et al., Letter of Intent to Construct a nuPRISM Detector in the J-PARC Neutrino Beamline, arXiv:1412.3086.

[25] JSNS2 collaboration, M. Harada et al., Proposal: A Search for Sterile Neutrino at J-PARC Materials and Life Science Experimental Facility, arXiv:1310 .1437.

[26] BoreXINO collaboration, G. Bellini et al., SOX: Short distance neutrino Oscillations with BoreXino, JHEP 1308 (2013) 038, [arXiv: 1304 . 7721].

[27] V. Barinov, V. Gavrin, D. Gorbunov and T. Ibragimova, BEST sensitivity to O(1) eV sterile neutrino, Phys. Rev. D93 (2016) 073002, [arXiv:1602.03826].

[28] M. Abs et al., IsoDAR@KamLAND: A Conceptual Design Report for the Technical Facility, arXiv:1511.05130.

[29] E. Ciuffoli, J. Evslin and F. Zhao, Neutrino Physics with Accelerator Driven Subcritical Reactors, JHEP 01 (2016) 004, [arXiv: 1509.03494$].$

[30] DANSS collaboration, I. Alekseev et al., DANSS: Detector of the reactor AntiNeutrino based on Solid Scintillator, JINST 11 (2016) P11011, [arXiv: 1606.02896$].$

[31] NEOS collaboration, B. R. Kim et al., Development and Mass Production of a Mixture of LAB-and DIN-based Gadolinium-loaded Liquid Scintillator for the NEOS Short-baseline Neutrino Experiment, arXiv:1511.05551.

[32] NEUTRINO-4 collaboration, A. P. Serebrov et al., NEUTRINO-4 experiment: preparations for search for sterile neutrino at 100 MW reactor SM-3 at 6-12 meters, arXiv: 1205.2955.

[33] PROSPECT collaboration, J. Ashenfelter et al., The PROSPECT Physics Program, J. Phys. G43 (2016) 113001, [arXiv:1512.02202].

[34] SoLID collaboration, N. Ryder, First results of the deployment of a SoLid detector module at the SCK-CEN BR2 reactor, PoS EPS-HEP2015 (2015) 071, [arXiv: 1510 . 07835].

[35] STEREO collaboration, V. Helaine, Sterile neutrino search at the ILL nuclear reactor: the STEREO experiment, arXiv:1604.08877.

[36] S. Mertens, Absolute neutrino masses, Talk presented at TAUP 2015, 7-11 September 2015, Torino, Italy (2015) .

[37] S. N. Axani et al., KPipe: a decisive test for muon neutrino disappearance, arXiv:1510.06994. 